\title{
The Reliability of the Prowler in High School Male Football Players
}

\author{
Granite Tano ${ }^{1}$, Alan Bishop ${ }^{2}$, Mike Climstein ${ }^{3}$ and Mark DeBeliso ${ }^{1}$ \\ 1. Department of Physical Education and Human Performance, Southern Utah University, Cedar City, UT 84720, USA \\ 2. Athletics Department, Utah State University, Logan, UT 84322, USA \\ 3. Exercise Health \& Performance Faculty Research Group, Faculty of Health Sciences, The University of Sydney, NSW, Australia
}

\begin{abstract}
Horizontal plane muscular power output is important in sports such as football especially for down lineman who must engage an opponent and push them for a number of yards. The Prowler resistance sled is a commonly used apparatus that aids in the development of horizontal plane muscular power output. However, there is limited documentation regarding the Prowler's role as a strength and conditioning training modality as well as its potential use as an assessment tool for measuring horizontal plane muscular power output. Hence, the purpose of this study was to assess the reliability of the Prowler sled push in High School male football players. High School male football players $(n=16)$ performed two trials of the Prowler sled push 91 kilograms $(200$ pounds) for a distance of 9.1 meters (10 yards). Each trial was timed with a handheld stop watch with a 2-3 minute rest period between trials. The trial 1 and 2 scores were $5.14 \pm 0.95$ and $5.15 \pm 0.88$ seconds respectively. The interclass and intraclass reliability coefficients were $r=0.93$ and $\mathrm{ICC}=0.93$. The standard error of the measure was $\mathrm{SE}_{\mathrm{m}}=0.25$ seconds with $90 \%$ confidence limits of $\mathrm{U}_{\mathrm{L}}: 0.36, \mathrm{~L}_{\mathrm{L}}: 0.20$. The mean difference between trials was $0.01 \pm 0.36$ seconds $\left(90 \%\right.$ confidence limits of $\left.\mathrm{U}_{\mathrm{L}}: 0.17, \mathrm{~L}_{\mathrm{L}}:-0.14\right)$. Within the parameters of this study, the Prowler sled push is a highly reliable field test for measuring horizontal plane muscular power output.
\end{abstract}

Key words: Reliability, Prowler, football, high school.

\section{Introduction}

The ability to improve speed and acceleration is an important athletic quality, which is crucial in a number of team (rugby union, football, soccer, hockey) and individual sports (sprinting, long jump, skating). The incorporation of sled training as part of strength and power training regimes is now common practice with various sports integrating its use for improvements in speed which is well documented in the literature $[1,2]$. Although initial research has focused upon the benefits of sled training on improving speed [1], subsequent studies have investigated the optimal load [3-5], biomechanical analyses of sprinting kinematics (technique, stride length, contact time) $[6,7]$ and establishing coefficients of friction to determine the

Corresponding author: Mark DeBeliso, Ph.D., professor, research fields: orthopedic biomechanics, mechanics and metabolics of sport movements and work-tasks, strength training for all walks of life, and master's athletes. amount of work performed during the activity [8]. These studies have primarily focused upon sled pulling, whereas investigations into sled pushing are quite limited and have focused upon either bob-skeleton athletes [9], strongman athletes [10] or EMG muscle activation [11].

Many sports require a participant to engage an opponent (or sport implement) and push forward in the horizontal plane. This includes football down linemen, wrestlers, mixed martial arts, rugby athletes, bobsledders, as well as certain Crossfit and strongman events. Sled training, where the athlete pushes a loaded sled in the horizontal plane, closely mimics the horizontal plane power requirements of the aforementioned sports by removing momentum and forcing the athlete to overcome inertia. Sled pushing in the horizontal plane, should be beneficial with respect to transferring muscular strength and power that was developed during traditional strength and conditioning 
protocols which include squats (back and front) and Olympic lift variations that occur primarily in the vertical plane with simultaneous bilateral leg engagement. Further, sled pushing aids in sport specific motor skill development within a horizontal plane due to total body engagement of the hands, arms, shoulder girdle, trunk, and lower extremity musculature during the push in a closed chain unilateral leg propulsion manner. One important differentiation of sled training from traditional weight training is that it has a very minimal eccentric component [12] which is highly beneficial as most muscular damage incurred from training is associated with the eccentric component [13].

Another important difference between sled towing and pushing has to do with forward trunk lean. Specifically, the issue that arises with sled pulling is that any forward trunk lean by the athlete causes forward movement of the sled, allowing momentum to aid in the pull. Conversely, a sled push requires overcoming inertia with each step, and thus will give a more accurate measure of horizontal plane muscular power output. Essentially, there is no way to "cheat" the exercise and makes the sled push a potential assessment tool to measure horizontal plane power output.

The Prowler is a commercially available resistance sled (Fig. 1) that has two sets of handles that enables

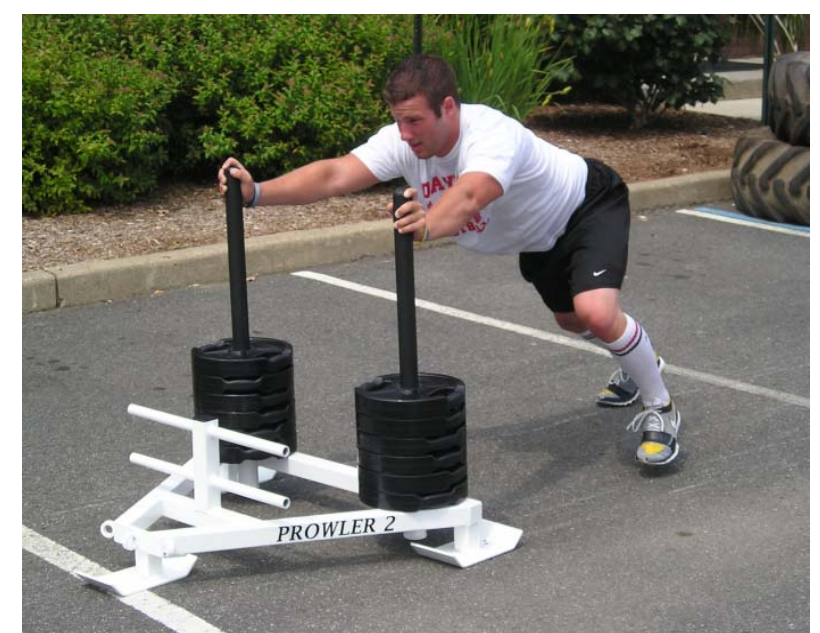

Fig. 1 Prowler resistance sled (total mass 91 kilograms pushed for 9.1 meters). the athlete to push the sled horizontally from either a low or high position. Fig. 1 demonstrates an athlete pushing the sled from the high handle position.

Given the training utility, specificity, and ease of use, the question arises as to if the Prowler might also serve as a reliable method of assessing horizontal plant power output in athletes. Hence, the purpose of this study was to determine the reliability of the Prowler sled push in a population of High School male football players.

\section{Methods}

\subsection{Participants}

A convenience sample of High School male football players were recruited and later volunteered for the study. Permission to conduct the study was obtained through a University Institutional Review Board. Each participant and parent provided written consent prior to engaging in the study.

\subsection{Procedures}

There was one testing session that included a warmup period consisting of the dynamic movements performed for a distance of 9.1 meters (10 yards). The participants performed one warm up movement one way $\&$ switched to another movement on the way back. The movements were: high knees, butt kickers, frog jumps, cherry pickers, lateral slide, karaokes, back pedal, and finished up with a sprint $(\approx 10$ minutes).

Following one practice trial, each participant performed two maximal effort trials of the Prowler sled push (Fig. 1). The Prowler was loaded with Olympic weights such that the total combined weight was 91 kilograms (200 pounds Prowler plus weights). The loaded Prowler was vertical handle pushed [12] by the participants for a total of 9.1 meters in a direct linear path. The trials were recorded with a hand held stop watch (100th second) and separated by a 2-3 minutes rest period. The same researcher timed all of the trials. The trials were conducted on the teams grass turfed practice field (dry \& recently cut) and the participants 
wore their cleated footwear. The testing session was conducted in late November immediately following the football offseason. All of the participants had previous experience performing the Prowler sled push.

\subsection{Statistical Analysis}

A combination of statistical approaches were used to determine the test-retest reliability of the Prowler sled push (trial 1 vs. trial 2). Interclass (r) and intraclass reliability coefficients (ICC) were calculated. The standard error of the measure $\left(\mathrm{SE}_{\mathrm{m}}\right)$ was also calculated. Scatter and Bland-Altman plots were constructed in order to explore uniformity of error. Trial data was Log-transformed in order to assess typical error as expressed as a coefficient of variation percent (CV\%). Further, 90\% confidence limits were calculated for the aforementioned reliability statistics. Statistics were calculated in Excel with a spreadsheet prepared by Hopkins [14]. This particular reliability analysis is identical to that reported in White et al. [15].

\section{Results}

Sixteen High School male football players participated in the study. The average age, height, and body mass of the participants is presented in Table 1 . All of the participants completed both test trials of the Prowler sled push without incident.

The reliability analysis is based one session of test re-test scores $(n=16)$. The mean of trial 1 and trial 2 scores from both sessions were $5.14 \pm 0.95$ and $5.15 \pm$ 0.88 seconds respectively, with a mean difference between trials of $0.01 \pm 0.36$ seconds. Fig. 2 is a scatter plot of trial 1 and 2 Prowler sled push scores while Fig. 3 is a Bland-Altman plot comparing the trial average scores versus the difference scores. The scatter plot suggests a linear relationship between the trial scores. The Bland-Altman plot demonstrates that only 1 of 16 data pairs exceeded the $95 \%$ limits of agreement [16]. Neither the scatter plot nor the Bland-Altman plot suggested bias or heteroscedasticity (non-uniform error). Of the 16 pairs of Prowler sled push scores, 7 were slower during trial 2 , and 9 were faster during trial 2.

Table 2 provides the reliability statistics with $90 \%$ confidence limits $\left(\mathrm{U}_{\mathrm{L}}, \mathrm{L}_{\mathrm{L}}\right)$. The intraclass reliability coefficient was ICC $=0.93(0.97,0.82)$, which is considered "above average acceptable" [17]. The interclass reliability coefficient was $\mathrm{r}=0.93(0.97$, 0.82 ), which is considered very high [18]. The standard error of the measure was $\mathrm{SE}_{\mathrm{m}}=0.25(0.36,0.20)$ seconds. While the trial data did not exhibit non-uniform

Table 1 Participant descriptive information.

\begin{tabular}{lll}
\hline Age (years) & Height $(\mathrm{cm})$ & Mass $(\mathrm{kg})$ \\
\hline $16.3 \pm 1.1$ & $177.2 \pm 6.7$ & $83.1 \pm 18.0$ \\
\hline
\end{tabular}

High School Male Football players, $N=16($ mean \pm sd).

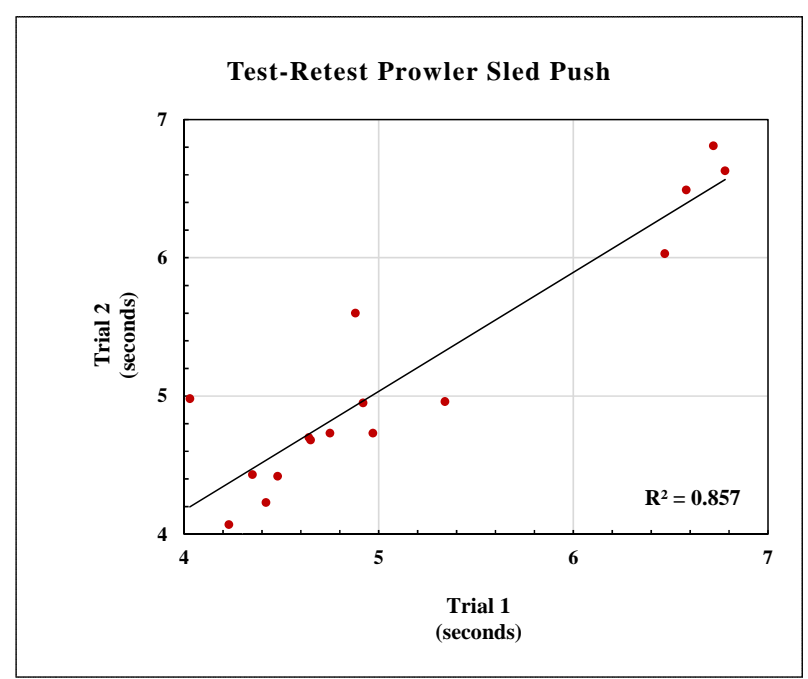

Fig. 2 Scatter plot of Trial 1 and Trial 2 Prowler scores.

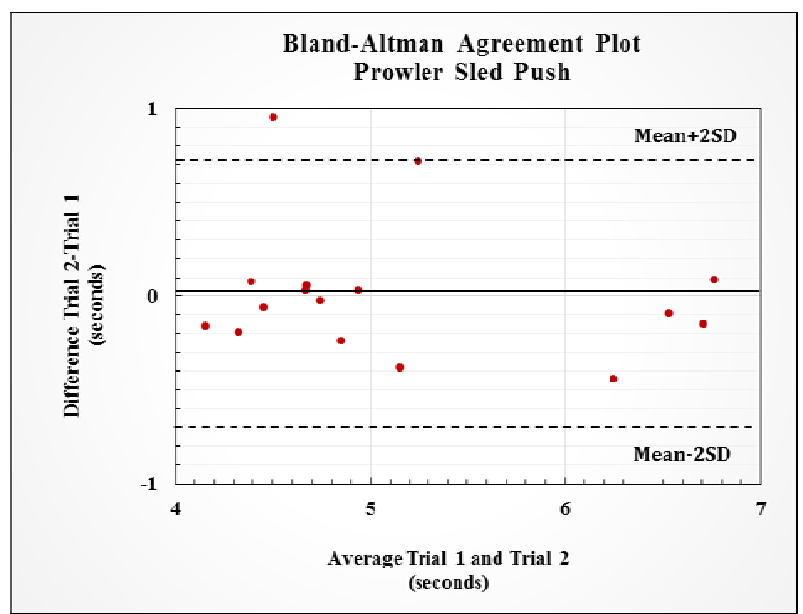

Fig. 3 Bland-Altman plot of Prowler Trial scores. 
Prowler sled push scores.

The trials were essentially split in that of the 16 participants 7 had faster trial one scores and 9 had faster trial two scores. As such, it would appear that fatigue did not impact the trial scores. Which would indicate that the rest periods between trials, the one practice trial, and the warmup selected for this study were appropriate.

The participants in this study were familiar with the Prowler and the particular pushing mechanics used in the current study. This familiarity likely added to the extremely reliable results noted in the current study. Future studies should include participants that are as familiar with the Prowler and focus on different sport/gender populations. Likewise, additional efforts should focus on different lengths of the push distance and load placed on the Prowler sled.

The load placed on the Prowler in the current study was selected as it was close to the average body weight of the participants (as well as the body weight of an opponent football player). College and professional football players are in general much larger in body size than high school players and would likely be better served by training/assessing with higher loads placed on the Prowler. Research regarding optimal loads for sled towing have focused on percentage of body mass (5, 12.5, and 20\%) [4]. Clearly the sled towing loads used for enhancing speed development would be too low for the purpose of developing horizontal plane power output of the sort described in the introduction.

A key point to keep in mind when using the Prowler sled push as a measure of horizontal plane power out is the horizontal plane resistive load of the sled. The resistive load of the sled is equal to the total weight of the loaded sled multiplied by the coefficient of friction between the sled ground surface and the ground surface covering (grass turf in this case). This study used a field of grass that was recently cut and dry. To assure reliable trials with the Prowler sled push test one must make sure that the turf conditions (height \& moisture) as well outside temperature are relatively the same on test dates. This precautionary note is due to the fact that the coefficient of friction of the grass turf can vary due to temperature, moisture and grass length.

Another point worthy of discussing has to do with the actual value of collecting a second trial of Prowler sled push scores. Generally speaking, individuals typically have better scores during subsequent repeated trials of a physical test due to some type of learning (in the absence of appreciable fatigue). Since that was not the case with the second trial of Prowler sled push scores, and given that the average difference in trial scores was 0.01 seconds, then why conduct two trials if one is looking for max performance? Is it possible that you only need one trial of Prowler sled push scores to establish horizontal plane power output in this population? Conversely, given the ease of collecting two trials and in the absence of fatigue on trial two scores, why not collect two trials of Prowler sled push scores to establish horizontal plane power output?

\section{Conclusions}

Within the parameters of this study, the Prowler sled push scores demonstrated a very high degree of reliability and should be considered a reliable test for measuring horizontal plane power output in High School male football players. The Prowler resistance sled is a versatile training implement with a high degree of task specificity and ease of use. Further, the Prowler sled push, when used to assess horizontal plane power output, is an easily administered test which is specific to a number of sport tasks. The authors recommend its use to physical educators and youth sport coaches who work with this population.

\section{Conflict Statement}

None of the authors have any known conflict of interest with the manufacturer of the Prowler or otherwise.

\section{References}

[1] Zafeiridis, A., Saraslanidis, P., Manou, V., Ioakimidis, P., Dipla, K., and Kellis, S. 2005. "The Effects of Resisted 
Sled-Pulling Sprint Training on Acceleration and Maximum Speed Performance." Journal of Sports Medicine and Physical Fitness 45 (3): 284-90.

[2] Clark, K. P., Stearne, D. J., Walts, C. T., and Miller, A. D. 2010. "The Longitudinal Effects of Resisted Sprint Training Using Weighted Sleds vs. Weighted Vests." Journal of Strength and Conditioning Research 24 (12): 3287-95.

[3] Alcaraz, P. E., Palao, J. M., and Elvira, J. L. 2009. "Determining the Optimal Load for Resisted Sprint Training with Sled Towing." Journal of Strength \& Conditioning Research 23 (2): 480-5.

[4] Bachero-Mena, B., and Gonza' lez-Badillo, J. J. 2014. "Effects of Resisted Sprint Training on Acceleration with Three Different Loads Accounting for 5, 12.5, and 20\% of Body Mass." Journal of Strength and Conditioning Research 28 (10): 2954-60.

[5] Naoki, K., Newton, R. U., Naruhiro, H., and Kazunori, N. 2014. "Effects of Weighted Sled Towing with Heavy versus Light Load on Sprint Acceleration Ability." Journal of Strength \& Conditioning Research 28 (10): 2738-45.

[6] Maulder, P. S., Bradshaw, E. J., and Keogh, J. W. 2008. "Kinematic Alterations Due to Different Loading Schemes in Early Acceleration Sprint Performance from Starting Blocks." Journal of Strength and Conditioning Research 22 (6): 1992-2002.

[7] Alcaraz, P. E., Elvira, J. L., and Palao, J. M. 2014. "Kinematic, Strength, and Stiffness Adaptations After a Short-Term Sled Towing Training in Athletes." Scandinavian Journal of Medicine \& Science in Sports 24 (2): 279-90.

[8] Andre, M. J., Fry, A. C., Bradford, L. A., and Buhr, K. W. 2013. "Determination of Friction and Pulling Forces during a Weighted Sled Pull." Journal of Strength and Conditioning Research 27 (5): 1175-8.

[9] Cook, C., Holdcroft, D., Drawer, S., and Kilduff, L. P. 2013. "Designing a Warm-up Protocol for Elite Bob-Skeleton Athletes." International Journal of Sports Physiology and Performance 8 (2): 213-5.
[10] Berning, J. M., Adams, K. J., Climstein, M., and Stamford, B. A. 2007. "Metabolic Demands of "Junkyard" Training: Pushing and Pulling a Motor Vehicle." Journal of Strength and Conditioning Research 21 (3): 853-6.

[11] Maddigan, M. E., Button, D. C., and Behm, D. G. 2014. "Lower-limb and Trunk Muscle Activation with Back Squats and Weighted Sled Apparatus." Journal of Strength \& Conditioning Research 28 (12): 3346-53.

[12] Jenkins, N. M. D., and Palmer, T. 2012. "Implement Training for Concentric-Based Muscle Actions." Strength \& Conditioning Journal 34 (2): 1-7.

[13] Clarkson, P. M., and Hubal, M. J. 2002. "Exercise-Induced Muscle Damage in Humans." American Journal of Physical Medicine and Rehabilitation 81 (11): S52-69.

[14] Hopkins, W. G. 2007. "Understanding Statistics by Using Spreadsheets to Generate and Analyze Samples." Sportscience 11: 23-36.

[15] White, K., DeBeliso, M., Sevene, T., and Adams, K. J. 2015. "The Reliability of the 300-Yard Shuttle Run in High School Girls Basketball Players." Journal of Sports Science 3 (5): 214-8.

[16] Bland, J. M., and Altman, D. G. 1986. "Statistical Methods for Assessing Agreement between Two Methods of Clinical Measurement." Lancet 8: 307-10.

[17] Baumgartner, T. A., Jackson, A. S., Mahar, M. T., and Rowe, D. A. 2007. Measurements for Evaluation in Physical Education and Exercise Science (8th ed.). New York, NY: McGraw Hill.

[18] Safrit, M. J., and Wood, T. M. 1995. Introduction to Measurement in Physical Education and Exercise (3rd ed.). St. Louis, MO: Mosby.

[19] Hopkins, W. G. 2013. A new view of statistics. Internet Society for Sport Science. Accessed July 4, 2016. http://www.sportsci.org/resource/stats/.

[20] Hoffman, J. 2006. Norms for Fitness, Performance, and Health. Champaign. IL: Human Kinetics.

[21] Bruton, A, Conway, J. H., and Holgate, S. T. 2000. "Reliability: What Is It and How Is It Measured?" Physiotherapy 86 (2): 94-9. 